Supplement of

\title{
Hygroscopic properties and cloud condensation nuclei activation of limonene-derived organosulfates and their mixtures with ammonium sulfate
}

\author{
A. M. K. Hansen et al. \\ Correspondence to: N. L. Prisle (nonne.prisle@ helsinki.fi)
}

The copyright of individual parts of the supplement might differ from the CC-BY 3.0 licence. 


\section{Supplementary information}

\section{Limonene organosulfates of MW $250 \mathrm{Da}$}
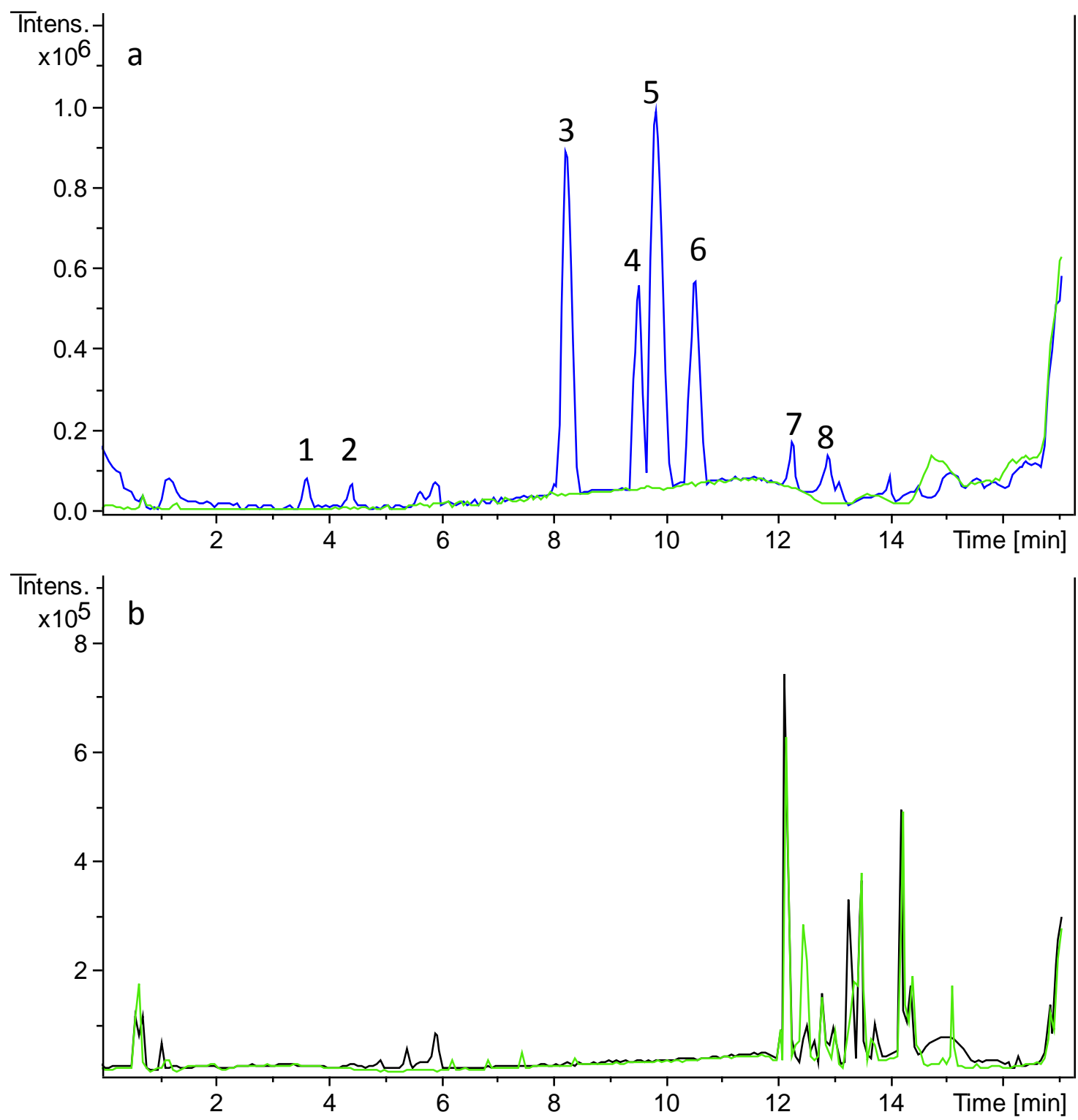

Figure 1: Base peak chromatograms of the purified limonene OS250 sample (blue) and a blank sample (green) using negative ionisation analysis (a) and positive ionisation analysis (b). peak 1: and 2: OS 286 (m/z 267.09), peak 3 to 6 : OS 250 (m/z 249.08) and peak 7 and 8: OS $384(\mathrm{~m} / \mathrm{z} 383.19)$.

Figure 1 shows base peak chromatograms from UHPLC-ESI-q-TOF-MS analysis of the purified organosulfate 250 sample using negative (a) and positive (b) ionisation. The purified organosulfate 250 sample does not show any additional compounds from the positive ionisation analysis and only 
a small signal from an organosulfate with MW 268 (peak 1 and 2) and an organosulfate with MW 384 (peak 7 and 8) is observed.

In Table 1 below the chemical formulas (based on suggestions from smart formula in the program “data analysis" from Burker Daltonics) and suggested structures for the observed organosulfates are given.

\begin{tabular}{|c|c|c|c|c|c|}
\hline & $\mathbf{m} / \mathbf{z}$ & RT [min] & msms fragments & Molecular formula & Suggested structure \\
\hline OS 268 & 267.09 & $4.4 ; 5.9$ & $96.96,79.95$ & $\mathrm{C}_{10} \mathrm{H}_{19} \mathrm{O}_{6} \mathrm{~S}$ & \\
\hline OS 250 & 249.08 & $\begin{array}{c}8.2 ; 9.5 \\
10.3\end{array}$ & 95.95 & $\mathrm{C}_{10} \mathrm{H}_{17} \mathrm{O}_{5} \mathrm{~S}$ & \\
\hline OS 384 & 383.19 & 15.4 & $\begin{array}{c}158.94,116.92 \\
96.96\end{array}$ & $\mathrm{C}_{20} \mathrm{H}_{31} \mathrm{O}_{5} \mathrm{~S}$ & \\
\hline
\end{tabular}

Table 1: Information on the organosulfates observed in the purified limonene organosulfate sample. Molecular formulas are suggested based on smart formula analysis in the program "data analysis" from Burker Daltonics. 


\section{CCN Calibration}

During measurements with the CCNc, the supersaturation (SS) was entered to be in the range of 0.1 to $1.3 \%$ increasing in steps of $0.1 \%$. Measurement of ammonium sulfate (AS) hence resulted in 13 appurtenant critical particle diameters (d50). The 13 measured d50-values for AS was used to correct the SS in the CCNc by calibration with theoretical SS for ammonium sulfate, using the Köhler equation (Seinfeld and Pandis, 2006):

$S S\left(D_{p}\right)=\left(\exp \left(\frac{4 M_{w} \sigma_{w}}{R T \rho_{w} D_{p}}-\frac{6 n_{s} M_{w}}{\pi \rho_{w} D_{p}^{3}}\right)-1\right) \cdot 100 \%$

where $\mathrm{M}_{\mathrm{w}}$ is the molecular weight of water $(18.0153 \mathrm{~g} / \mathrm{mol}), \sigma_{\mathrm{w}}$ is the surface tension of water $\left(0.07286 \mathrm{~J} / \mathrm{m}^{2}\right), \mathrm{R}$ is the gas constant $(8.314 \mathrm{~J} / \mathrm{kg} \mathrm{K}), \mathrm{T}$ is the temperature $(298.15 \mathrm{~K}), \rho_{\mathrm{w}}$ is the density of water $\left(997.1 \mathrm{~kg} / \mathrm{m}^{3}\right)$, Dp is the droplet diameter, and $\mathrm{ns}$ is given below:

$n_{s}=\frac{v \pi d_{s}^{3} \rho_{s}}{6 M_{s}}$

where $v$ is the van Hoff factor, $\rho_{\mathrm{s}}$ is the density of AS $\left(1770 \mathrm{~kg} / \mathrm{m}^{3}\right), \mathrm{d}_{\mathrm{s}}$ is the diameter of the dry AS particle (here the measured d50) and $\mathrm{M}_{\mathrm{s}}$ is the molecular weight of AS (132.1395 g/mol).

The van Hoff factor was calculated with the following parametrisation as suggested by (Prisle, 2006):

$v=-0.002 * c_{A S}^{5}+0.032 c_{A S}^{4}-0.197 C_{A S}^{3}+0.5997 C_{A S}^{2}-0.8431 c_{A S}+2.3552$

For each of the 13 measured d50-vlaues a Köhler curve was modelled by setting $\mathrm{d}_{\mathrm{s}}$ to $\mathrm{d} 50$ and calculating SS while increasing $\mathrm{D}_{\mathrm{p}}$ in steps of 1.01. The critical supersaturation (SS50) was then found as the top of the Köhler curve. Figure 2 shows the modelled Köhler curve for ds $=133.34$ $\mathrm{nm}$. 


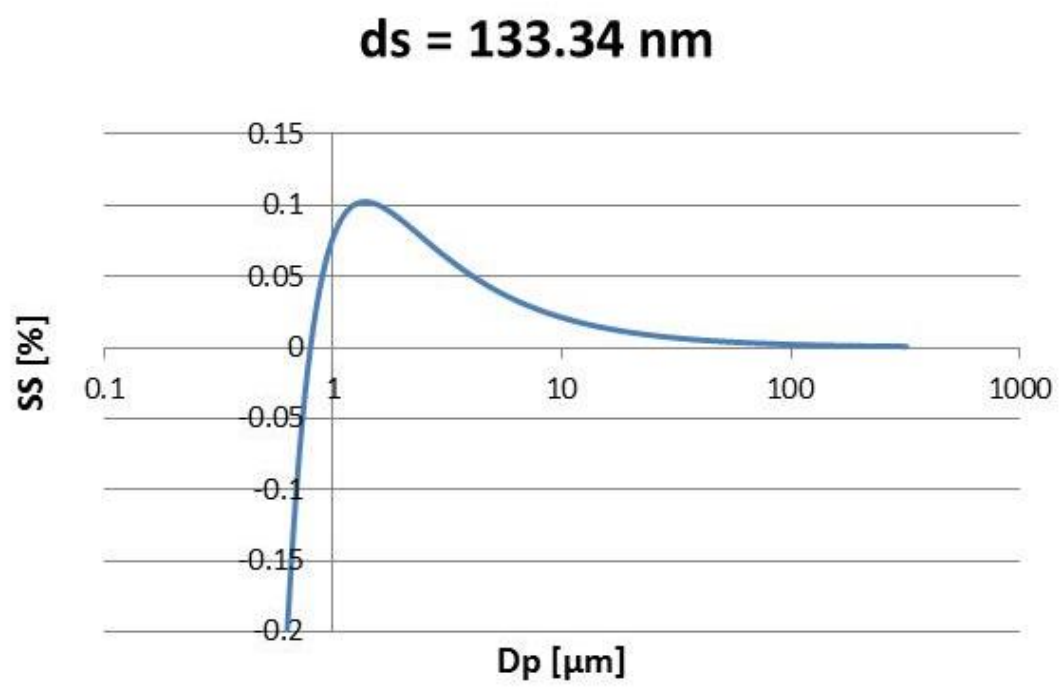

Figure 2: Köhler curve for AS with a dry particle diameter, ds, of $133.34 \mathrm{~nm}$.

The theoretical SS50-values found from the Kóhler curves were then plotted against the SS entered in the $\mathrm{CCNc}$ (Figure 3) and the following regression line was obtained, where the uncertainties were found using the linear regression approach:

$S S_{\text {corrected }}=0.686( \pm 0.006) \cdot S S_{\text {entered in the } C C N c}+0.031(0.005)$

The SS in the CCNC was corrected using the regression line and the entered SS, resulting in corrected SS in the range of 0.099 to $0.922 \%$.

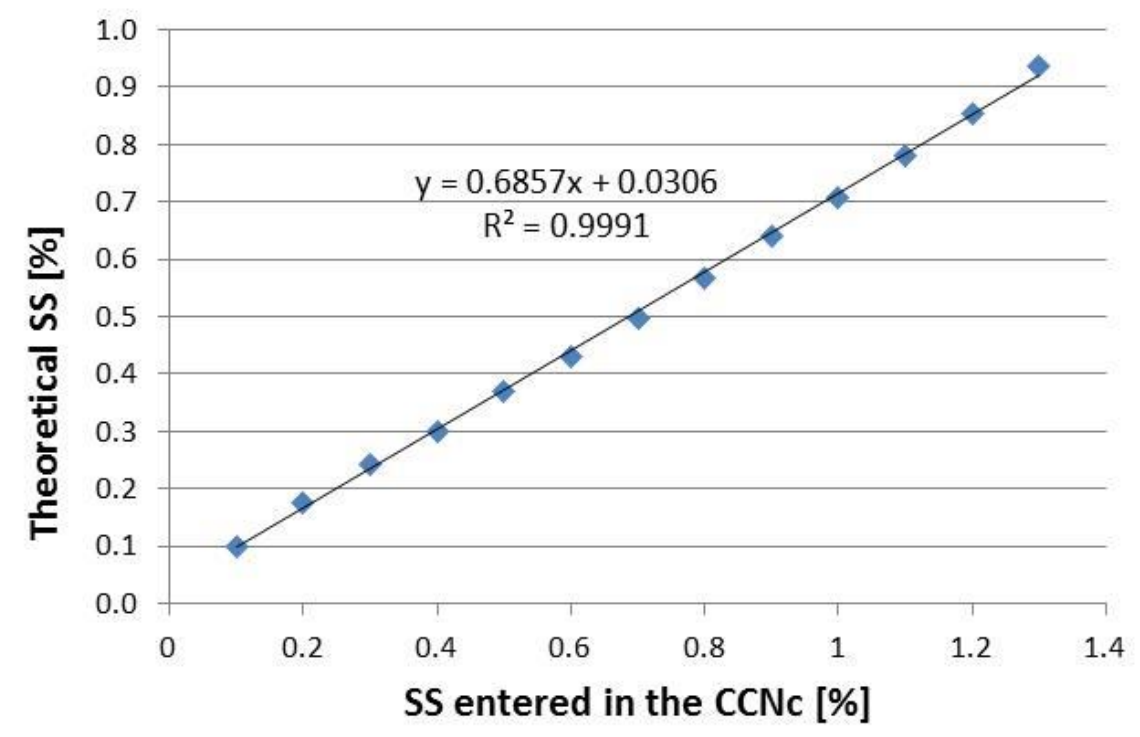

Figure 3: Calibration curve for correction of SS in the CCNC. 


\section{Bulk-to-surface Partitioning}

The thermodynamic approach to calculate surfactant bulk-to-surface partitioning in cloud droplets is based on the original work of Li et al. (1998) who considered the effect of surfactant partitioning on droplet solution surface tension, and Sorjamaa et al. (2004) who also accounted for surfactant effect on the water activity (or mole fraction). The single component model is presented by Prisle et al. (2008) and the model for surfactant-salt mixtures, which is used in the current calculations, is presented by Prisle et al. (2010). The main idea in these calculations is that when the surfactant partitions to the droplet surface, it is effectively removed from the bulk solution which determines the thermodynamic properties of the droplet (e.g., surface tension and water activity). The approach to calculate surfactant partitioning is based on the Gibbs adsorption equation or isotherm (Gibbs et al., 1928), which can be given in the following form:

$\sum_{i} n_{i}^{T} R T d \ln \left(a_{i}^{B}\right)+A d \sigma^{B}=0$

where $\mathrm{n}$ is number concentration, $\mathrm{a}$ is activity, $\mathrm{A}$ is droplet surface area, $\sigma$ is droplet solution surface tension, $\mathrm{R}$ is the gas constant, $\mathrm{T}$ is temperature, and $\mathrm{d}$ refers to differential. Subscript $\mathrm{i}$ cover all liquid phase species. Superscripts T and B refer to total and bulk phases, respectively. The total number concentrations are known from the given dry particle composition and droplet diameter $($ total $=$ bulk + surface $)$. In the current approach, all species are allowed to partition between bulk and surface phases so that the surface partitioning of the surfactant is replaced by equal volume of surface depletion of water and other non-surfactant solutes (pseudo-binary solution composed of one surfactant and water + non-surfactant solute mixture). As a result, the total surface volume is zero and bulk volume is equal to the total droplet volume. With these model considerations, the adsorption equation has only one unknown (bulk concentration of the surfactant).

Solving the adsorption equation requires an activity coefficient model ( $a_{i}^{B}=\gamma_{i}^{B} x_{i}^{B}$, where $\gamma$ is the activity coefficient and $x$ is mole fraction) and a surface tension parameterization. Also, these must be thermodynamically consistent. Since activity coefficient models are not available for the current mixtures, those were set to unity, i.e., an ideal solution is expected. The ideal solution assumption is always thermodynamically consistent, but there are some requirements for the surface tension parameterization. It has to be continuous and the differential must become zero at the infinitely dilute surfactant solution. The Szyskowski equation (Eq. 1 in the main text) is thermodynamically 
consistent and the gradient (e.g. $\frac{d \sigma^{B}}{d n^{B}}$ ) is negative after the infinite dilution limit, which results in positive surfactant adsorption at all non-zero surfactant concentrations. Although surface tensions of single surfactant solutions (with or without added salt) are often in good agreement with the Szyskowski equation, this is not the case for multicomponent surfactant solutions or surfactant solutions with surface active impurities. In addition, different solubility values and chemical equilibrium transitions (e.g. protonation/deprotonation) can result in unexpected changes to surface tension of multicomponent solutions with respect to total solute concentration. Clear deviations from the smooth surface tension behavior were also observed here (Fig. 3 in the main text). Although the current surface tension parameterization may not be the most accurate one, it is thermodynamically consistent and continuous over all surfactant and salt concentrations.

During the model calculations, both dry particle size and composition is fixed and the droplet diameter is given. As described above, bulk phase concentrations are found numerically by solving the adsorption equation. When the model is used to simulate hygroscopic growth, the simulations give the correct equilibrium RH (based on Eq. 9 in the main text). For the CCN experiments, the simulations give the equilibrium supersaturation (also based on Eq. 9 in the main text), but the critical supersaturation is found numerically by varying droplet size until the maximum equilibrium supersaturation is found.

With a few simplifications (e.g., fixed type of surface tension parameterization and ideal solutions) an analytical solution can be found for the surfactant partitioning equilibrium (Topping, 2010; Raatikainen and Laaksonen, 2011). Later these computationally efficient analytical equations have been used in studies assessing the large scale effects of surfactants (e.g. Prisle et al., 2012; Topping and McFiggans, 2012). These analytical equations have also been used to extend the $\kappa$-Köhler theory to account for surfactant partitioning (Petters and Kreidenweis, 2013). Although the analytical equations are based on additional approximations and assumptions, their predictions are very similar to those of the full thermodynamic model (e.g. Raatikainen and Laaksonen, 2011). The model has been used here, because it can account for the effect of the added ammonium sulfate salt on the droplet solution surface tension. 


\section{References}

Gibbs, J. W., Bumstead, H. A., Van Name, R. G., and Longley, W. R.: The Collected Works of J. Willard Gibbs: Thermodynamics, Longmans, Green and Co., 1928.

Li, Z. D., Williams, A. L., and Rood, M. J.: Influence of soluble surfactant properties on the activation of aerosol particles containing inorganic solute, J Atmos Sci, 55, 1859-1866, Doi 10.1175/1520-0469(1998)055<1859:Iosspo>2.0.Co;2, 1998.

Petters, M. D., and Kreidenweis, S. M.: A single parameter representation of hygroscopic growth and cloud condensation nucleus activity - Part 3: Including surfactant partitioning, Atmos Chem Phys, 13, 1081-1091, 10.5194/acp-13-1081-2013, 2013.

Prisle, N. L.: Cloud Condensation Nuclei Properties of Organic Aerosol Particles: Effects of Acid Dissociation and Surfactant Partitioning, M.Sc. Thesis, Department of Chemistry, Faculty of Science, University of Copenhagen, Copenhagen, Denmark, 2006.

Prisle, N. L., Raatikainen, T., Sorjamaa, R., Svenningsson, B., Laaksonen, A., and Bilde, M.: Surfactant partitioning in cloud droplet activation: a study of C8, C10, C12 and C14 normal fatty acid sodium salts, Tellus B, Chem. Phys. Meteorol., 60, 416-431, 10.1111/j.16000889.2008.00352.x, 2008.

Prisle, N. L., Raatikainen, T., Laaksonen, A., and Bilde, M.: Surfactants in cloud droplet activation: mixed organic-inorganic particles, Atmos Chem Phys, 10, 5663-5683, 10.5194/acp-105663-2010, 2010.

Prisle, N. L., Asmi, A., Topping, D., Partanen, A. I., Romakkaniemi, S., Dal Maso, M., Kulmala, M., Laaksonen, A., Lehtinen, K. E. J., McFiggans, G., and Kokkola, H.: Surfactant effects in global simulations of cloud droplet activation, Geophys Res Lett, 39, Artn L05802, 10.1029/2011g1050467, 2012.

Raatikainen, T., and Laaksonen, A.: A simplified treatment of surfactant effects on cloud drop activation, Geosci Model Dev, 4, 107-116, 10.5194/gmd-4-107-2011, 2011.

Seinfeld, J. H., and Pandis, S. N.: Atmospheric Chemistry and Physics: From Air Pollution to Climate Change, 2. ed ed., Wiley, Hoboken, New Jersey, 2006.

Sorjamaa, R., Svenningsson, B., Raatikainen, T., Henning, S., Bilde, M., and Laaksonen, A.: The role of surfactants in Kohler theory reconsidered, Atmos Chem Phys, 4, 2107-2117, 2004.

Topping, D.: An analytical solution to calculate bulk mole fractions for any number of components in aerosol droplets after considering partitioning to a surface layer, Geosci Model Dev, 3, 635-642, 10.5194/gmd-3-635-2010, 2010.

Topping, D. O., and McFiggans, G.: Tight coupling of particle size, number and composition in atmospheric cloud droplet activation, Atmos Chem Phys, 12, 3253-3260, 10.5194/acp-123253-2012, 2012. 